\title{
Glinical Analysis of Hypertrophic Cardiomyopathy which Evolved into Dilated Phase during Long-term Follow-up
}

\author{
Kagumi DoI, MD, Genji ToDs, MD, \\ Ivan Iliev Iliev, MD, Motonobu Hayano, MD, \\ and Katsusuke YANO, MD
}

\section{SUMMary}

The aim of the present study was to analyze the incidence, clinical features and prognosis of patients with hypertrophic cardiomyopathy (HCM) which evolved into dilated phase HCM. The medical records of 43 patients with HCM followed up for at least 10 years were analyzed retrospectively. The patients were divided into two groups: group A consisting of patients with dilated-phase HCM defined by a left ventricular end diastolic dimension (LVDD) of $55 \mathrm{~mm}$ or more and a left ventricular ejection fraction (LVEF) of less than $50 \%$ obtained by echocardiography, and group B, consisting of patients with HCM that did not evolve into dilated phase HCM. During the mean follow-up of 16.7 years, 10 patients $(23.3 \%)$ evolved into dilated phase HCM (group A) while the remaining 33 patients $(76.7 \%$ ) did not (group B). Ventricular tachycardia (VT) occurred in 7 of the 10 patients $(70.0 \%)$ in group $A$ and in 5 of the 33 patients $(15.2 \%)$ in group $B(p<0.001)$. An increase in LVDD and decreases in LVEF and SVI + RV5 in the electrocardiogram were observed during the early phase of the follow-up period in group A, while these changes were gradual in group B. Cardiac death occurred in $5(50.0 \%)$ of the 10 patients in group A and in $2(6.1 \%)$ of the 33 patients in group B $(p<0.001)$. In conclusion, dilated-phase HCM is characterized by decreases in LVEF and SV1 + RV5 and an increase in LVDD during the early phase of follow-up period, and is associated with an increased incidence of VT and a poor prognosis. (Jpn Heart J 1999; 40: 579-587)

Key words: Hypertrophic cardiomyopathy, Dilated phase, Long-term follow-up

$\mathrm{H}$

YPERTROPHIC cardiomyopathy (HCM) has been reported to have marked heterogencity in cardiac morphology and natural course. Although left ventricular hypertrophy $(\mathrm{LVH})$ with normal systolic function and diastolic dysfunction is considered to be the fundamental feature of the disease,

From the Third Department of Internal Medicine, Nagasaki University School of Medicine, Nagasaki, Japan. Address for correspondence: Kagumi Doi, MD, the Third Department of Internal Mcdicine, Nagasaki University, 1-7-1, Sakamoto-cho, Nagasaki City, Nagasaki 852-8501, Japan.

Received for publication July 8, 1999.

Revised and accepted August 25, 1999. 
on a few occasions a different pathway of cvolution of the disease has been reported. ${ }^{1)}$ It includes systolic dysfunction, dilatation of the left ventricle (LV) and a decrease in LV wall thickness. This type of natural cvolution of HCM has been termed the dilated phase of hypertrophic cardiomyopathy (dilated-phase HCM). ${ }^{2}$ However, strict criteria for dilated-phase HCM have not yet been proposed, and the pathophysiology of this process is not fully understood. The patterns of changes in hypertrophy in the natural course of HCM arc varied, and nobody can currently predict the development of dilated-phase HCM in an individual patient. In an attempt to contribute to a better understanding of this process, we conducted the present study with the purpose of cvaluating the incidence, clinical characteristics and prognosis of patients with HCM which evolved into dilatedphase HCM during a long term follow-up period.

\section{Patients and Methods}

The medical records of 43 patients (28 males, 15 females) with HCM who had been followed up for at least 10 years were analyzed. The diagnosis of HCM was made on the basis of clinical and clectrocardiographic criteria and was confirmed by the presence of typical echocardiographic or angiographic findings, or both. Cardiac catheterization data were available for 37 patients. Patients with coronary artery discasc, hypertension leading to $\mathrm{LVH}$, or any cvidence of secondary cardiomyopathy were excluded. Patients with systolic dysfunction or LV dilatation at the time of the first examination were also excluded. The mean age of the 43 patients at the time of the first examination was $43.3 \pm 14.9$ years, ranging from 13 to 78 years. The mean follow-up period was $16.7 \pm 4.2$ years, ranging from 10 to 26 years. The patients were divided into two groups: group A consisting of those who evolved into dilated-phase HCM which was characterized by a left ventricular end diastolic dimension (LVDD) of $55 \mathrm{~mm}$ or more and a left ventricular ejection fraction (LVEF) of less than $50 \%$, and group B consisting of those who did not evolve into dilated phase HCM with a LVDD of less than $55 \mathrm{~mm}$ and an LVEF of $50 \%$ or more, evaluated using M-mode echocardiography (Teichholtz method) over the follow-up period. Patients were examined biennially. A chest roentgenogram, twelve lead elcctrocardiogram (ECG) and M-mode echocardiogram were obtaincd during a follow-up visit. The pattern of the hypertrophic changes of the left ventricle was determined in every patient upon entry into the study and during the follow-up period, using two dimensional echocardiography. Patients with asymmetrical septal hypertrophy (ASH, an intraventricular scptum (IVS) to left ventricular posterior wall (LVPW) ratio greater than 1.3) with echocardiography were diagnosed as ASH type HCM. Non-ASH patients were echocardiographically classified into apical, mid- 
ventricular, lateral, posterior, and diffuse type HCM according to the site of hypertrophy. Diffuse type HCM was defined as diffuse LVH with IVS/ LVPW $<1.3$. Clinical findings and the results of electrocardiography, echocardiography and chest roentgenogram upon entry into the study and during the follow-up period in patients with dilated-phase HCM were compared with those of patients without LV dilatation and systolic dysfunction. In order to evaluate the ECG changes during the follow-up period, we measured the sum of the amplitudes of $\mathrm{S}$ wave in lead V1 and $\mathrm{R}$ wavc in lead V5 (SV1 + RV5) in twelve lead ECG in the first examination and during the follow-up period.

\section{Results}

During the follow-up period, LV dilatation with systolic dysfunction occurred in 10 patients $(23.3 \%)$ while these changes were not observed in the remaining 33 patients $(76.7 \%)$.

Clinical characteristics of the patients (Table I): The mean age and mean duration of follow-up were not significantly different between the two groups.

Table I. Clinical Characteristics of the Patients

\begin{tabular}{llll}
\hline & Group A & Group B & \\
\hline Number $(\%)$ & $10(23.3 \%)$ & $33(76.7 \%)$ & \\
Age at first examination & $41.5 \pm 12.2$ & $43.8 \pm 15.6$ & $\mathrm{~ns}$ \\
Age at last examination & $59.5 \pm 9.6$ & $59.7 \pm 15.7$ & $\mathrm{~ns}$ \\
Follow up (years) & $17.6 \pm 5.1$ & $16.4 \pm 6.1$ & $\mathrm{~ns}$ \\
Familial HCM & $3(30.0 \%)$ & $13(39.4 \%)$ & $\mathrm{ns}$ \\
Ventricular tachycardia & $7(70.0 \%)$ & $5(15.2 \%)$ & \\
& SVT 4 & SVT 1 & 0.001 \\
Atrial fibrillation & NSVT 3 & NSVT 4 & \\
& $5(50.0 \%)$ & $14(42.4 \%)$ & \\
Peripheral embolism & Paf 1 & Paf 7 & $\mathrm{~ns}$ \\
\hline
\end{tabular}

SVT = sustained ventricular tachycardia; NSVI = non-sustained vent ricular tachycardia; $\mathrm{Pa}$ [ = paroxysmal atrial fibrillation; Caf $=$ chronic atrial fibrillation.

Table II. Patterns of Left Ventricular Hypertrophy at First Examination

\begin{tabular}{lll}
\hline & Group A & Group B \\
\hline ASH & $7(70.0 \%)$ & $15(4.5 .5 \%)$ \\
Apical & $0(0 \%)$ & $8(24.2 \%)$ \\
Diffuse & $2(20.0 \%)$ & $4(12.1 \%)$ \\
Posterior & $1(10.0 \%)$ & $0(0 \%)$ \\
Mid-ventricular & $0(0 \%)$ & $1(3.0 \%)$ \\
Lateral & $0(0 \%)$ & $1(3.0 \%)$ \\
\hline
\end{tabular}

ASH = asymmetric apical hypertrophy. 
$\mathrm{mV}$

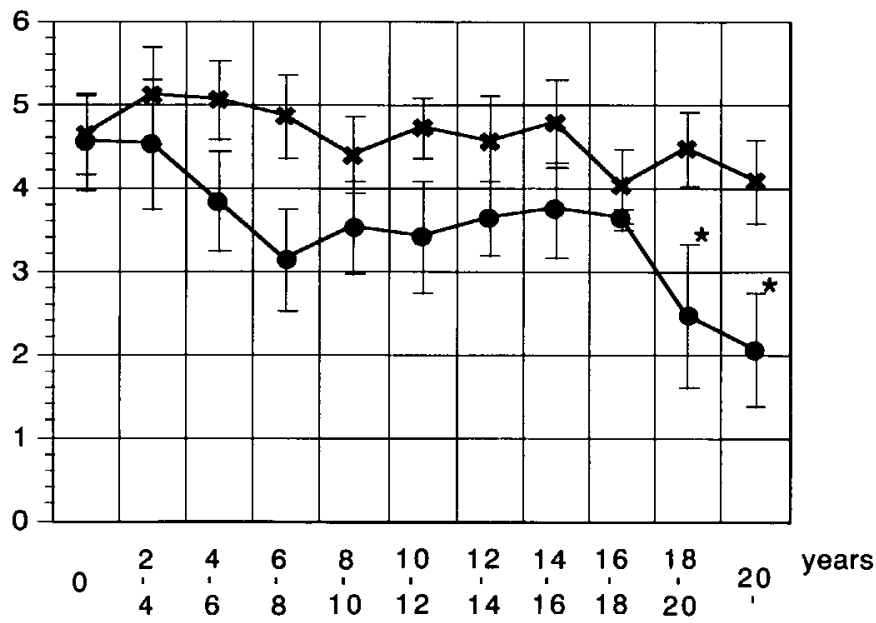

Figure 1. Time course of sum of the amplitude of $S$ wave in lead $\mathrm{Vl}$ and $\mathrm{R}$ wave in lead V5 (SV1 + RV5) in groups A and B. Abscissa shows the years of follow-up. Ordinate shows the amplitude of SVI + RV5. Values are plotted as the mean $\pm \mathrm{SE}$. " $X$ " represents group B patients and " group A patients. $*<<0.05$. See text for details.

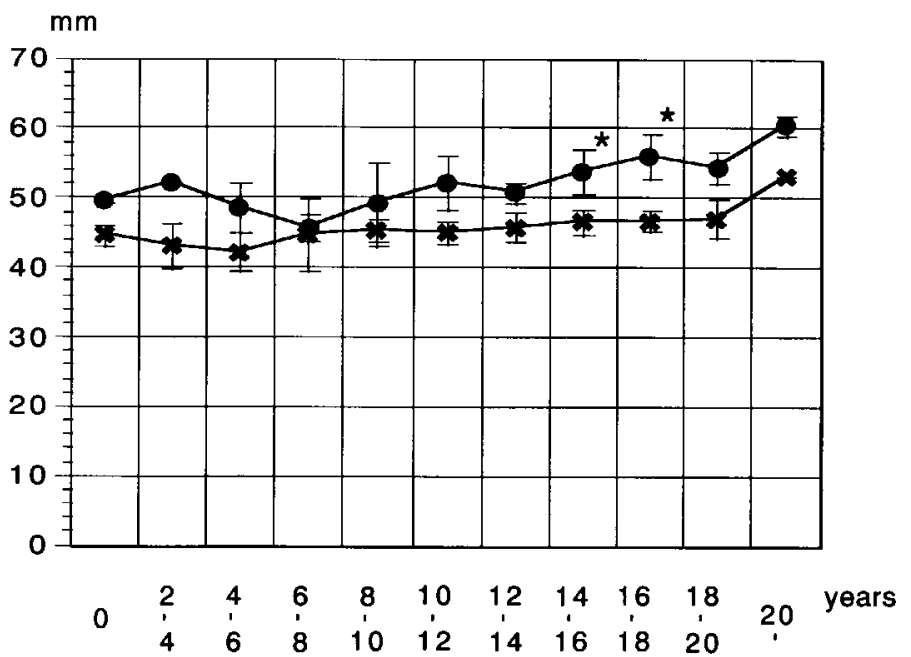

Figure 2. Time course of left ventricular end diastolic dimension during follow-up period in groups $A$ and $B$. Values are plotted as the mean \pm SE. " $X$ " represents group B patients and " group A patients. ${ }^{*} p<0.05$. See text for details. 


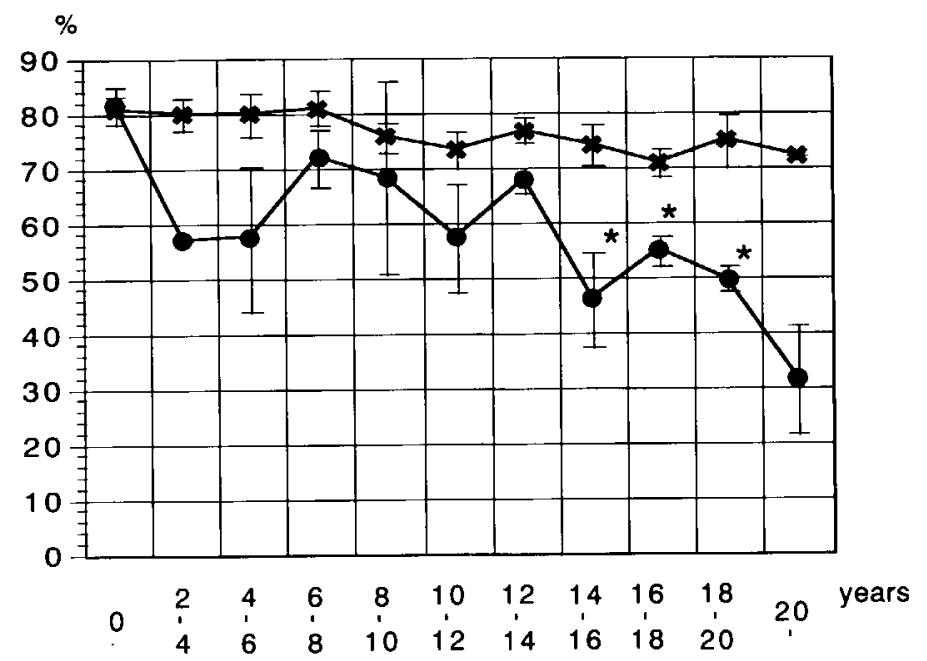

Figure 3. Time course of changes in left ventricular cjection fraction during followup period in groups $A$ and $B$. Values are plotted as the mean \pm SE. " $X$ " represents group B patients and " $O$ " group A patients. * $p<0.05$. See text for details.

Ventricular tachycardia occurred in 7 of $10(70.0 \%)$ group A patients and in 5 of $33(15.2 \%)$ group B patients $(p<0.001)$. The incidences of familial HCM, hypertension, atrial fibrillation and peripheral embolism were similar in the two groups.

Chest roentgenographic, electrocardiographic, and echocardiographic findings during the first examination: The mean cardio-thoracic ratio (CTR) was $52.8 \pm 3.4 \%$ in group $\mathrm{A}$ and $52.2 \pm 5.4 \%$ in Group B. There was no significant difference in CTR between the two groups. Mean SV1+RV5 was $4.24 \pm 1.43 \mathrm{mV}$ in group $\mathrm{A}$ and $4.96 \pm 2.17 \mathrm{mV}$ in group $\mathrm{B}$ at first examination ( $p=n s$ ). The patterns of left ventricular hypertrophy at first examination in the two groups are shown in Table II. Asymmetric septal hypertrophy (ASH) was observed in 7 of the $10(70.0 \%)$ group A patients and in 13 of the $33(45.5 \%)$ group B patients $(p=0.191)$. The wall thicknesses of the intraventricular septum (IVS) and left ventricular posterior wall (LVPW) in the ASH cases in both groups were very similar (IVS $=20.0 \pm 4.5 \mathrm{~mm}$ in group $\mathrm{A}, 20.8 \pm 3.0 \mathrm{~mm}$ in group $\mathrm{B}$, $\mathrm{LVPW}=12.3 \pm 2.4 \mathrm{~mm}$ in group $\mathrm{A}, 12.5 \pm 2.1 \mathrm{~mm}$ in group $\mathrm{B}, p=\mathrm{ns})$. Apical hypertrophy was observed in 8 of the $33(24.2 \%)$ group B patients, but not in any group A patients $(p=n s)$. Obstruction of the left ventricular outflow tract (LVOT) was confirmed in 3 of the $10(30.0 \%)$ group A patients and in 9 of the $33(27.3 \%)$ group $B$ patients. The difference in the incidence of obstruction of LVOT between the two groups was not significant.

Mean LVDD was $46.4 \pm 3.7 \mathrm{~mm}$ in group A and $43.5 \pm 5.2 \mathrm{~mm}$ in group 
B. Mcan LVEF was $74.1 \pm 8.1 \%$ in group A and $80.3 \pm 7.2 \%$ in group B. Mean left atrial dimension (LAD) was $40.5 \pm 3.9 \mathrm{~mm}$ in group $A$ and $36.4 \pm 6.3 \mathrm{~mm}$ in group B. The LVDD, LVEF and LAD values in group A were not significantly different from those in group $B$.

Electrocardiographic changes during the follow up period: The changes in $\mathrm{SV} 1+\mathrm{RV} 5$ over time are shown in Figure 1. As shown in Figure 1, the decrease in SV1 + RV5 in group A began during the early phase and a further decrease in $\mathrm{SVl}+\mathrm{RV} 5$ was observed in the late phase of the follow-up period. A gradual decrease in SVI + RV5 was observed during the follow-up period in group B patients. The level of ST depression and the amplitude of negative $\mathrm{T}$ wave in group $\Lambda$ also decreased gradually during the follow-up period.

Echocardiographic changes during the follow up period: The changes in LVDD over time in the two groups are shown in Figure 2. There was an increase in LVDD beginning during the early phase of the follow-up period in group A. However, the increase in LVDD was gradual in group B.

The changes in LVEF over time in the two groups are shown in Figure 3. A decrcase in LVEF in group A patients began during the early phase of the follow-up period and progressed with time. On the other hand, the decrease in LVEF in group B patients was gradual and only slight.

Prognosis: Five of the $10(50.0 \%)$ group A patients and 6 of the $33(18.2 \%)$ group B patients $(p<0.001)$ died. Cardiac death occurred in 5 of the $10(50.0 \%)$ group A patients and in 2 of the $33(6.1 \%)$ group B $(p<0.001)$ paticnts. Three of the group A patients died of congestive heart failure while the remaining 2 died suddenly, probably due to ventricular arrhythmias. One group B patient died of congestive heart failure and the other of ventricular arrhythmia. These patients in group B had a rapid increase in LVDD and a decrease in LVEF, so at the time of death they were close to meeting the criteria of dilated phase HCM. The cause of death in the remaining 4 group $B$ patients was respiratory failure in 1, malignant tumors in 2 and unknown in 1.

\section{Discussion}

In the present study, we compared retrospectively the clinical findings and clinical courses in patients with HCM who evolved into dilated phase HCM with those in patients without significant LV dilatation or LV systolic dysfunction during a mean follow-up period of 16.7 years. LV dilatation and LV systolic dysfunction occurred in $10(23.3 \%$ ) of the 43 paticnts (annual incidence $1.40 \%$ ). Incidence of dilated-phase HCM: Frank and Braunwald" reported cardiac enlargement in 20 of 126 patients with $\mathrm{HCM}$ (total incidence 16\%, annual incidence $1.3 \%$ ) during a 12 year follow-up period. Progression to left ventricular 
dilatation in 2 (total incidence $4 \%$, annual incidence $1 \%$ ) of 50 patients with HCM followed up for 4 years was reported by ten Cate et al..$^{3)}$ Fighali $^{4)}$ followed up 62 patients with HCM for an average of 8 years and reported the development of segmental or generalized left ventricular hypokinesia in 5 (total incidence $8 \%$, annual incidence $1 \%$ ). Scilcr ${ }^{5}$ reported progression to left ventricular dilatation in 20 patients (total incidence 14\%, annual incidence $1.36 \%$ ) during a mean follow-up of 10.3 years in 139 patients with HCM. Thus, the frequency of dilated-phase HCM reported in previous studies is about $10 \%$, with an annual incidence of approximately $1-1.3 \%$. In the present study, we observed a total incidence of $23.3 \%$ and an annual incidence of $1.3 \%$ during a mean follow-up of 16.7 years. The total incidence of dilated-phase HCM in the present study was higher than those of previous reports, most likely because of the longer follow-up period.

Clinical characteristics of patients with dilated-phase HCM at first examination: We compared the clinical findings at first examination in the HCM patients who evolved into dilated phase HCM during the follow-up period with those in HCM patients who did not. There were no significant differences in the patterns of left ventricular hypertrophy, LVEF, LVDD, LAD, CTR or SV1 + RV5 at first examination between the two groups. Previous studics also failed to find any differences in clinical findings upon entry into the study between HCM patients who evolved into dilated phase and HCM patients who did not. ${ }^{1-6)}$

Evolution of the electrocardiogram and echocardiogram changes in dilatedphase HCM: Toshima $e$ al.$^{2}{ }^{2}$ concluded from their analysis of 32 patients with dilated-phase HCM that the electrocardiographic features of dilated-phase HCM include the disappearance of LV hypertrophy, a decrease in $\mathrm{R}$ wave amplitude in chest lead, the appearance of abnormal $Q$ waves, and the appearance of conduction disorder, atrial premature contractions and ventricular premature contractions. In the present study, a decrcase in SVI + RV5 in the twelve lead electrocardiogram was obscrved during the early phase of the follow-up pcriod in all dilated-phase HCM patients. This change was accompanied by gradual decrcases in the level of ST depression and the amplitude of the negative ' $\mathrm{T}$ wave. Therefore this finding can be thought to represent electrocardiographically the decrease in LVH. Ventricular tachycardia occurred in $7(70.0 \%)$ of the 10 patients with dilated-phase HCM. The decrease in SV1 + RV5 and the appcarance of ventricular tachycardia may be important findings which suggest the evolvement of HCM into the dilated phasc. Moreover, Toshima et al. suggested that the echocardiographic features of dilated-phase HCM include a decrcase in LVEF and an increase in LVDD. In the present study, a decrease in LVEF and an increase in LVDD during early phase of the follow-up period was observed in 
group A, but not in group B. ASH was observed in $7(70.0 \%)$ of the group A patients. The wall thicknesses of IVS and LVPW in the ASH cases in both groups were almost identical at first examination. Therefore, in our study, it is not possible to conclude that $\mathrm{ASH}$ type $\mathrm{HCM}$ evolves more frequently into dilated phase.

Prognosis of dilated-phase HCM: Since both systolic and diastolic dysfunction are observed in patients with dilated-phase HCM, the treatment is usually difficult. Cannan et al. ${ }^{7}$ reported that during a mean follow-up of 7.7 years in 37 patients with $\mathrm{HCM}$, the $1-$ and 5-year survival rates were $95 \%$ and $92 \%$, respectively, while the annual risk of cardiac death was $0.7 \%$. Spirito et al. ${ }^{6)}$ reported that during a mean follow-up of 3.6 years in 67 patients with HCM, the clinical course was poor in 8 who evolved into dilated phase, including 4 who died of congestive cardiac failure and 1 who underwent cardiac transplantation. In the present study, cardiac death occurred in $5(50.0 \%)$ of the 10 patients with dilatedphase HCM, including 3 who died of congestive heart failure and 2 who died suddenly, most likely due to ventricular arrhythmias. Our data suggest that acute exaccrbation of heart failure is rare in HCM patients, while slow progression of dilation and systolic dysfunction of LV is a common clinical course. Therefore, the prognosis of HCM patients who evolved into dilated phase may have been poor.

Study limitations: One major limitation of the present study is that a retrospcctive analysis was conducted. Another was the unavailability of information concerning medication. Therefore, the influence of medication on the clinical course and prognosis was not taken into consideration.

Conclusion: In the present study, evolution of hypertrophic cardiomyopathy into the dilated phase of hypertrophic cardiomyopathy (dilated-phase HCM) was observed to begin with systolic dysfunction and dilation of the left ventricle and diminution of ventricular voltage in precordial clectrocardiogram during the early phase of the follow-up period, all of which progressed with time. Furthermore, patients with dilated-phase HCM had a high incidence of VT and a poorer prognosis compared with HCM which did not cvolve into dilated-phase.

\section{References}

1. Frank S, Braunwald E. Idiopathic hypertrophic subaortic stenosis: clinical analysis of 126 patients with cmphasis on the natural history. Circulation 1968; 37: 759-88.

2. Toshima H, Adachi K. HCM evolved into dilated HCM. Kokyu to Junkan (Respiration and Circulation) 1988; 36: 597-608 (in Japancsc).

3. ten Cate FJ, Roelandt J. Progression to left ventricular dilation in patients with hypertrophic obstructive cardionyopathy. Am Heart J 1979; 97: 762-5.

4. Fighali S, Krajcer Z, Leachman RD. Progression of hypertrophic cardiomyopathy into a hypokinetic left ventricle: higher incidence in patients with midventricular obstruction. J $\Lambda \mathrm{m}$ Coll Cardiol 1987; 9: 
288-94.

5. Seiler C, Jenni R, Vassalli G, Turina M, Hess OM. Left ventricular chamber dilatation in hypcrtrophic cardiomyopathy: related variables and prognosis in patients with medical and surgical therapy. $\mathrm{Br}$ Heart J 1995; 74: 508-16.

6. Spirito P, Maron BJ, Bonow RO, Epstein SE. Occurrence and significance of progressive left ventricular wall thinning and relative cavity dilatation in hypertrophic cardiomyopathy. Am J Cardiol 1987; 59 123-9.

7. Cannan R, Reeder GS, Bailey KR. Natural history of hypertrophic cardiomyopathy: a populationbased study, 1976 through 1990. Circulation 1995; 92: 2488-94. 